\title{
Clinicopathologic Correlation of a Subretinal Proliferative Vitreoretinopathy Band in a Patient with Chronic Recurrent Retinal Detachment
}

\author{
Karen R. Brown Sander R. Dubovy Nidhi Relhan Harry W. Flynn Jr. \\ Department of Ophthalmology, Bascom Palmer Eye Institute, University of Miami, Miller \\ School of Medicine, Miami, FL, USA
}

\section{Keywords}

Proliferative vitreoretinopathy $\cdot$ Clothesline $\cdot$ Subretinal band

\begin{abstract}
Purpose: Proliferative vitreoretinopathy is a well-known cause of failure of retinal detachment surgery. The purpose of this case report is to illustrate the clinical occurrence and histopathology of a horizontal subretinal band ("clothesline" configuration) creating recurrent and persistent retinal detachment. Observations: A 67-year-old Hispanic female with diabetes type 2 and a history of retinal detachment surgery in the left eye (OS) presented with decreased vision OS. Best corrected visual acuity at this recent presentation was 20/80 OS. Clinical examination disclosed a recurrent inferior retinal detachment and a subretinal "clothesline" fibrotic band. Surgical removal of the subretinal band was performed. Histopathological evaluation of longitudinal and transverse sections of the band revealed a cable-like configuration composed predominantly of glial differentiation, RPE differentiation, and collagen, based on morphology and immunohistochemical staining. There was focal smooth muscle and neuroendocrine cell differentiation, as detected with smooth muscle actin (SMA) and S100 staining, respectively. Cross-sections demonstrated pigmented fibrocellular tissue with foci of cells staining positive for S100 and keratin peripherally around the tissue, suggestive of RPE differentiation. Scattered
\end{abstract}


foci of SMA-positive cells suggested mild myoblastic differentiation. Conclusions and Importance: This case report presents further information on the structure and orientation of the cellular components of subretinal band proliferative vitreoretinopathy. Cells suggestive of Müller cell differentiation compose the central aspect of the band, alongside collagen fibers. RPE differentiation is variably present peripherally in the band, likely reflective of proliferating RPE encircling the subretinal fibrous tissue. A mild amount of myofibroblastic differentiation was present within the band of tissue, correlating with the clinical findings of subretinal tissue contraction and localized retinal detachment.

(C) 2018 The Author(s)

Published by S. Karger AG, Basel

\section{Introduction}

Proliferative vitreoretinopathy is the most frequent cause of failure of retinal detachment surgery [1]. The composition of proliferative vitreoretinopathy includes glial, retinal pigment epithelial, and myofibroblastic differentiation [2-5]. Subretinal "napkin ring" configuration has also been described [6], as well as other directional membranes in localized quadrants of the retina. The purpose of the current report is to illustrate the clinicopathological correlation of a horizontal subretinal band ("clothesline" configuration) associated with recurrent and persistent retinal detachment.

\section{Case Report}

A 67-year-old Hispanic female with a history of type 2 diabetes mellitus presented with a 6-month history of recurrent visual loss in the left eye (OS). Two years prior to presentation, she had undergone scleral buckle, pars plana vitrectomy, internal drainage of subretinal fluid, fluid air exchange, endolaser, and $\mathrm{C}_{3} \mathrm{~F}_{8}$ gas in the OS. The patient was lost to follow-up until she returned with progressive visual loss, OS.

Her best-corrected visual acuity was $20 / 80$ OS and the clinical examination demonstrated the presence of recurrent inferior retinal detachment extending posterior to the scleral buckle. A striking subretinal fibrotic "clothesline" membrane extended horizontally from 4 o'clock to 8 o'clock (Fig. 1). Because of the visible retinal elevation by the subretinal band, surgical removal of the band was planned. In this patient, there were no visible epiretinal membranes or signs of retinal inflammation.

During a 23-gauge pars plana vitrectomy, a retinotomy was created with diathermy near the central portion of the subretinal fibrosis. ILM forceps were used to enter the subretinal space and to remove the subretinal band, which was submitted for histopathological examination. A fluid-air exchange was performed and internal drainage of subretinal fluid was accomplished, followed by endolaser around the retinotomy site as well as posterior to the scleral buckle inferiorly. Silicone oil was infused into the air-filled eye and a single suture was placed in the sclerotomy used for oil infusion. At the 4-month postoperative follow-up, the patient had retinal reattachment, but visual acuity remained 20/200 in the oil-filled OS.

Cross-sectional examination of the tissue revealed a cable-like configuration composed predominantly of glial differentiation, RPE differentiation and collagen, based on morphology and immunohistochemical staining with glial fibrillary acidic protein (GFAP), cytokeratin and S100, and Gomori trichrome immunohistochemical staining, respectively. The composition of the band consisted predominantly of cells positive for both GFAP and glutamine synthetase, suggestive of Müller cell differentiation. Gomori trichrome staining revealed collagen fibers 
arranged in a longitudinal fashion lengthwise within the band. There was pigment embedded within the tissue and foci of cells staining positive for $\mathrm{S} 100$ and keratin peripherally around the tissue, suggestive of RPE differentiation (Fig. 2a, b). Scattered foci of smooth muscle actinpositive cells suggested mild myoblastic differentiation.

\section{Discussion}

While immunohistochemistry cannot definitively identify the origin of the cells, it can demonstrate the morphologic characteristics of the cells present within the band. Cells suggestive of Müller cell differentiation compose the central aspect of the band, alongside collagen fibers. RPE differentiation is variably present peripherally in the band, likely reflective of proliferating RPE that encircled the subretinal fibrous tissue. A mild amount of myofibroblastic differentiation was present within the band of tissue, correlating with the clinical findings of subretinal tissue contraction and localized retinal detachment.

\section{Statement of Ethics}

Informed consent for the treatment was obtained from the patient. The manuscript conforms to the tenets of the Declaration of Helsinki and does not include any patient information or pictures which may reveal patient's identity.

\section{Disclosure Statement}

None of the authors have any potential conflicts of interest to disclose.

\section{Funding Sources}

Supported in part by the Florida Lions Eye Bank (Miami, Florida), the National Institute for Health (NIH) Center Core Grand P30EY014801 (Bethesda, Maryland), and Research to Prevent Blindness Unrestricted Grant (New York, New York) to the Department of Ophthalmology, Bascom Palmer Eye Institute, Miami, FL, USA.

\section{References}

1 Wallyn RH, Hilton GF. Subretinal fibrosis in retinal detachment. Arch Ophthalmol. 1979 Nov;97(11):2128-9.

2 Laqua H, Machemer R. Clinical-pathological correlation in massive periretinal proliferation. Am J Ophthalmol. 1975 Nov;80(5):913-29.

3 Van Horn DL, Aaberg TM, Machemer R, Fenzl R. Glial cell proliferation in human retinal detachment with massive periretinal proliferation. Am J Ophthalmol. 1977 Sep;84(3):383-93.

4 Constable IJ, Nagpal M. "Pathogenesis of proliferative vitreoretinopathy." Ryan's Retina. Elsevier, 2018; 2031-2051.

5 García-Arumí J, Corcostegui B, Tallada N. Subretinal membranes in proliferative vitreoretinopathy. An immunohistochemical study. Retina. 1992;12(3 Suppl):S55-9.

6 Tabandeh H, Callejo SA, Rosa RH Jr, Flynn HW Jr. Subretinal “napkin-ring” membrane in proliferative vitreoretinopathy. Arch Ophthalmol. 2000 Sep;118(9):1287-9. 


\section{Case Reports in Ophthalmology}

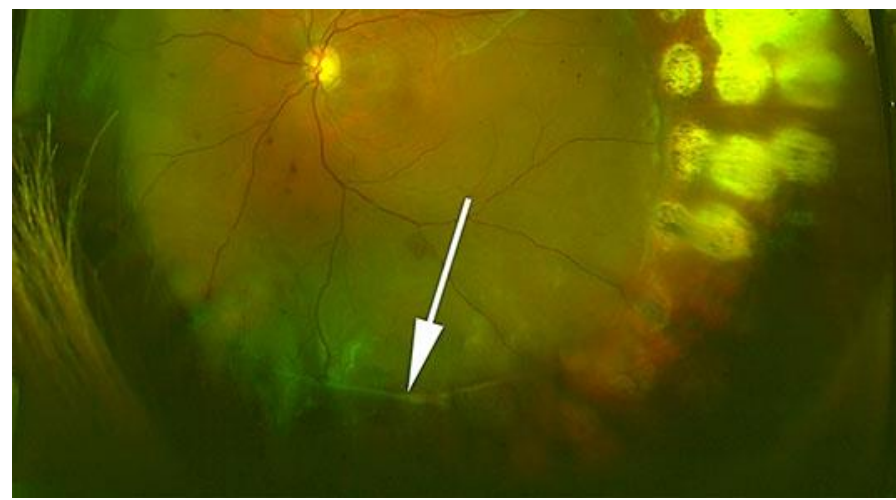

Fig. 1. Color fundus photograph of the left eye (preoperative): inferior retinal detachment with subretinal "clothesline" band evident inferiorly from 4 o'clock to 7 o'clock (arrow).

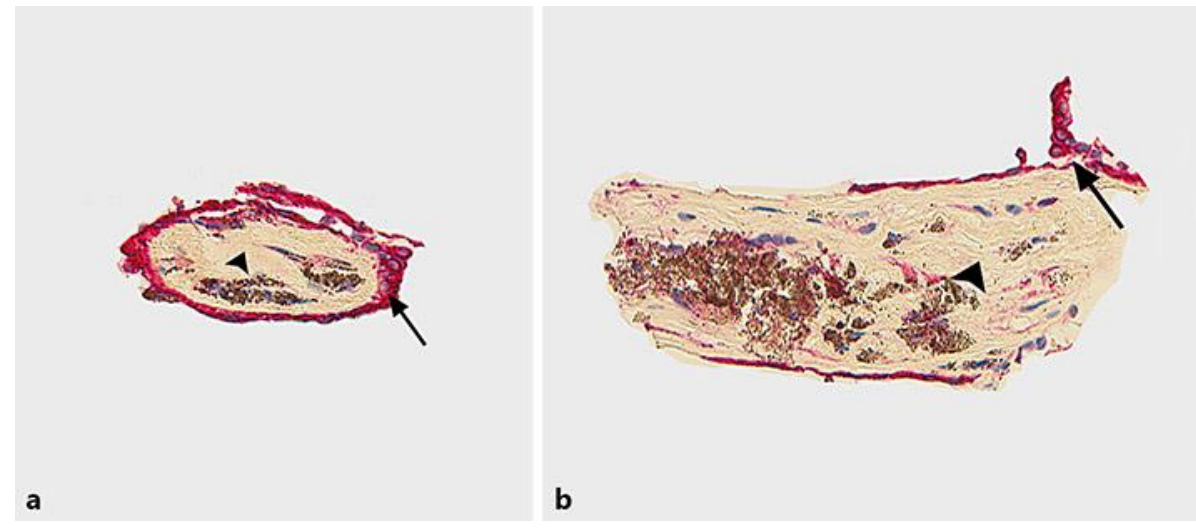

Fig. 2. a Cross-section of the subretinal band demonstrates collagen (arrowhead) and fibrocellular tissue, with cytokeratin staining (arrow), suggestive of retinal pigment epithelium peripherally in the band (cytokeratin with red chromogen, original magnification $\times 200$ ). $\mathbf{b}$ Longitudinal section of the subretinal band demonstrates collagen deposition (arrowhead), pigmented fibrocellular tissue, and cytokeratin-positive cells along the periphery (arrow), suggestive of RPE cell differentiation peripherally along the band (cytokeratin with red chromogen, original magnification $\times 200$ ). 